\title{
Comparative Evaluation of Probabilistic and Deterministic Tag Anti-collision Protocols for RFID Networks
}

\author{
Jihoon Choi and Wonjun Lee* \\ Division of Computer and Communication Engineering \\ College of Information and Communication \\ Korea University \\ wlee@korea.ac.kr
}

\begin{abstract}
Radio Frequency Identification (RFID) is an auto recognition system that consistis of a number of tags and readers. There could be multiple tags a reader should identify. Since a medium is shared by multiple tags, a collision occurs at the reader's side when two or more tags get transmitted simultaneously. Therefore, anti-collision mechanism that collaborately arbitrates collisions is required. Tag anti-collision schemes can be classified into two approaches according to the way to determine the point of transmission time: probabilistic approach and deterministic approach. These two approaches show dissimilar performance according to situations because of difference of process property. In this paper, we analyze the most advanced ever-existing tag anti-collision schemes and standards, and evaluate the performance of them with newly proposed metrics in various network situations.
\end{abstract}

\section{Introduction}

Radio Frequency Identification (RFID) is an auto recognition system that consists of a number of tags and readers. A RFID reader identifies objects by reading the data contained in tags [1]. The RFID reader communicates with tags through radio frequency, which is performed in a different manner of the barcode system in which a reader identifies a barcode through the light. Due to these characteristics, the RFID has wider range of identification of tags such that tags can be identified even when line of sight (LOS) does not get obtained. For such reasons, the RFID system has been spotlighted as the technology which can replace barcode system.

Tags can be classified into two types based on the existence of self electric power: active tag and passive tag. The active tag can transmit data without the aid of a reader because it has its own battery. It also has a more powerful memory than a passive tag. On the other hand, it is possible for a passive tag to transmit only when a reader is involved since it does not support self electric

\footnotetext{
* Corresponding author.
} 
power. A passive tag has constraints in functionality, but it has the distinct feature to the active tag: it is enough small to attach to an object easily. In this paper, we consider only passive tags.

There could be multiple tags a reader should identify. All the functionality tags should do is that they response with the data corresponding to the signal received from a reader. The communication between tags is impossible. Passive tags cannot make a decision of whether the channel is busy or not. A collision is occurred at the reader's side when two more tags get transmitted simultaneously. Therefore, the arbitrational mechanism is required. We call the protocol aiming to avoid collisions between a reader and tags anti-collision protocol. The anticollision protocol should have the following characteristics.

- A reader should identify all the tags within its range.

- The anti-collision algorithm should have a mechanism which is capable of verifying that all the tags are identified.

- It should minimize the time elapsed for the identification of tags. It lies on same line as reducing collisions. As the time which is required to identify tags increases, it is more difficult to identify objects moving fast.

Tag anti-collision schemes can be classified into two approaches according to the way to determine the point of transmission time: probabilistic approach and deterministic approach. In this paper, we introduce various anti-collision schemes including AQS and ABS, which we have suggested in our previous work. We also evaluate their performance. This paper is organized as follows. We give a detailed description about probabilistic and deterministic anti-collision schemes in section 2. Section 3 introduces adaptive tag anti-collision algorithms, and is followed by performance metrics and evaluation in section 5 and section 6 , respectively. Section 6 concludes the paper.

\section{Backgrounds}

Probabilistic tag anti-collision schemes are based on ALOHA. ALOHA is one of the basic medium access control mechanisms. In ALOHA, each tag generates a random number and waits for its transmission time according to the number chosen. If the data transmitted by a tag is not interfered by other data, the reader can identify the tag. A tag continues to do the same work after its transmission; generating a new random number and transmitting its own data after waiting for random amount of time. If during the interval two or more tags transmit, a collision occurs. In order to solve partial collision problems, transmission time is divided into discrete time intervals in the slotted ALOHA. All tags try to transmit their data after random back-off. If there are no partial collisions under the slotted ALOHA scheme, the slotted ALOHA doubles the channel utilization. A framed slotted ALOHA 22 3] 4, 5] 6] groups some slots into a frame. A frame has several transmission slots. The starting point of frame and slot is synchronized by a message of a reader. Tags determine their transmission slot when they receive a starting message from a reader. Transmission slot is set by random number generator in tags. 
The important factor which has influence on performance is the relation between the number of tags and random space, the maximum value of back-off timer. If the random space is larger than the number of tags in the reader's range, there can be many collision slots. On the other hand, if it is smaller than the number of tags, the frame may have too many idle slots. It is important to set suitable random space by predicting the number of tags.

According to the number of tag transmissions in a slot, slot can be divided into three types as follow:

- Readable slot: Exactly one tag transmits its data. The reader recognizes a tag successfully.

- Collided slot: More than two tags transmit their data. A tag collision occurs and the reader cannot recognize any tags.

- Idle slot: No tag transmits its data.

Under the frame slotted ALOHA schemes, the frame size means the size of random space. It is easy to change the frame size at each start of a frame. There exist many proposed schemes which improve the frame slotted ALOHA. The optimal frame size is the occasion when the number of tags and frame size are exactly the same. Many schemes that estimate the number of tags, using the number of readable slots, collision slots and idle slots, have been proposed. The symbol notions and their descriptions used thoughout this paper are summarized in Table 1.

Table 1. Notations

\begin{tabular}{|c|l|}
\hline symbol & description \\
\hline \hline$F$ & The frame size \\
$N_{t a g}$ & The estimated number of tags \\
$S$ & The number of readable slots \\
$C$ & The number of collided slots \\
$I$ & The number of idle slots \\
$S_{E X P}\left(F, N_{\text {tag }}\right)$ & The expected value of readable slots \\
& given frame size and the number of tags \\
$C_{E X P}\left(F, N_{\text {tag }}\right)$ & The expected value of collided slots \\
$I_{E X P}\left(F, N_{\text {tag }}\right)$ & given frame size and the number of tags \\
& The expected value of idle slots \\
given frame size and the number of tags
\end{tabular}

A readable slot which occurred in the previous frame is the one that contains only one tag. A collision slot has at least two tags. The lower bound of the number of tags can be estimated as Eq. (1).

$$
\text { Vogt } 1: N_{\text {tag }}=S+2 C
$$

In [4, the author proposed another way to estimate the number of tags. If we know the frame size and the number of tags, we can calculate the expected value 
of readable slots, idle slots and collided slots. By using Chebyshevs inequality, we can make tag estimation function as Eq. (2).

$$
\operatorname{Vogt2}: \text { est }_{f}=\min _{N_{\text {tag }}}\left|\left(\begin{array}{c}
S_{E X P}\left(F, N_{\text {tag }}\right) \\
C_{E X P}\left(F, N_{\text {tag }}\right) \\
I_{E X P}\left(F, N_{\text {tag }}\right)
\end{array}\right)-\left(\begin{array}{c}
S \\
C \\
I
\end{array}\right)\right|
$$

The probability mass function of the number of reacting tag in a slot can be obtained by using binomial distribution. By using these probabilities, we are able to calculate the collision ratio [5], which is a fraction of the number of collision slots to the frame size. The number of tags can be obtained by Eq. (3).

$$
D F S A: C_{\text {ratio }}=1-\left(1-\frac{1}{F}\right)^{N_{\text {tag }}}\left(1+\frac{N_{\text {tag }}}{F-1}\right)
$$

Actually the number of tags in collision slot can be more than two. We can get the expected number of tags in collision slot approximately 2.39 tags [6]. We can estimate the number of tags by using Eq. (4).

$$
\text { Zhen }: N_{\text {tag }}=S+2.39 C
$$

Under deterministic tag anti-collision schemes, a tag determines the point of transmission upon receiving a message from a reader and making a process from the message. A reader divides tags into two groups. The reader divides the one of them into two groups once again. It is required that reader is able to distinguish each divided group. The process of dividing tags is continued until a group contains only one tag. The fact that the number of tags in that group is one means that a reader can identify the tag successfully. The dividing process of a group is continued until a reader identifies all the tags.

The reader transmits a query to tags under the query tree (QT) scheme 7 . The query contains the prefix of the tag identification (ID) codes. Every tag in range of reader compares the query of reader with its ID codes and transmits its ID codes to the reader in case the result of comparing is true. This scheme uses a query of reader, prefix of tag ID Codes, to divide tags into two groups. Tags in one group transmit their ID codes to the reader. Tags in the other group wait for next query of the reader. The content of query is identifier of each group. The reader repeats to divide tags into two groups until the number of tags in a group is one. When the number of tags in a group is one, the reader successes to identify one tag. This identification process can be considered constructing a searching tree based tag ID Codes. The reader increases the length of the query until the identification cycle is finished. Fig. 1 shows an example of the identification process using the query tree protocol.

The binary tree (BT) scheme 3] uses the pseudo random number generator to divide tags into two groups. The counter variable in each tag is used for identifying each group. At the beginning of identification operation, the reader sends the message which notifies the start of cycle to tags. All tags received this message generate a random number 0 or 1 . Tags set their counter value by 




Fig. 1. An example of the identification process using the query tree protocol

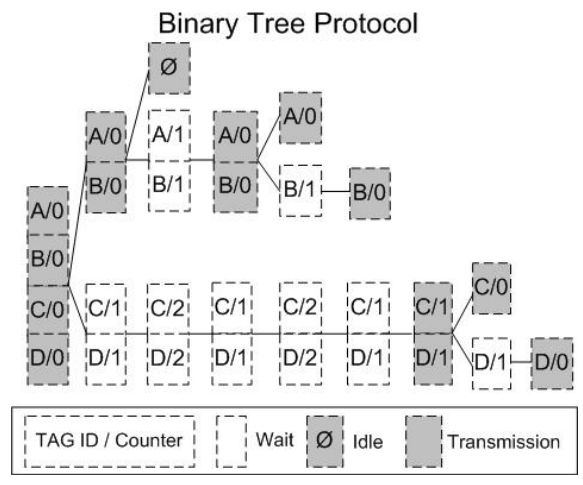

Fig. 2. An example of the identification process using the binary tree protocol

adding the random number. Tags are divided into two groups: one group has counter value of 0 . The other group has counter value of 1 .

The group with counter value of 0 tries to transmit and waits for the reply of the reader. If a collision occurs, tags which try to transmit in previous cycle are divided into two groups by using random number and tags which do not try to do increase the value of their counter by 1 . If there is no collision, all tags decrease the value of their counter by 1 . The tag identified successfully set the value of its counter to 0 and wait the start of frame message of the reader. Fig. 2 shows an example of the identification process using the binary tree protocol.

\section{Adaptive Splitting Schemes}

The main advantage of deterministic tag anti-collision scheme is that all tags in identification range of the reader can be identified. An identification cycle is a process that constructs a tree from root node to leaf nodes. When next identification cycle begins, information of tree in previous cycle is initialized. Following two schemes can improve the performance by using information of tree in previous cycle.

\subsection{Adaptive Query Splitting}

Adaptive Query splitting (AQS) 8] is our previous work and is the method that can reduce search space of current cycle by using the queries in leaf node of the tree which constructed in previous cycle. Searching from leaf node of the tree can diminish identification delay caused by query tree scheme which starts from root node of tree and can cover all possible search spaces.

The reader maintains not only the queue $\mathrm{Q}$ but also a candidate queue CQ. There are prefixes of tag ID codes in readable slots and idle slot from the previous 
identification cycle in CQ. At the start of the identification process, the reader initializes $\mathrm{Q}$ with the CQ and empties out CQ.

Fig. 3(a) shows the operation of the adaptive query splitting protocol. If the population of tags is same as previous identification cycle, no collision occurs in current cycle under AQS scheme. If there exists an incoming tags whose ID matches a prefix of readable nodes in previous cycle, it decreases the number of collision nodes to use prefixes of readable nodes. If a new tag of which ID does not match a prefix of a readable node in previous cycle is quickly identified with prefixes of idle nodes in previous cycle by the reader.

To prevent the increase of CQ which carries information of leaf nodes uses the query deletion process. The query deletion process is the process that merges queries which are same except last one bit to one query.

\subsection{Adaptive Binary Splitting}

One of our previous work, Adaptive Binary Splitting (ABS) 9], is the method that has tags remember their identification order in previous cycle by adding one more counter in tags. The tag maintains progressed-slot counter (PSC) and allocated-slot counter (ASC). PSC means the number of timeslots passed in an identification cycle. At the start of an identification cycle, PSC is initialized with 0 . In every readable slot, all tags increase their PSC by 1 . ASC determine whether a tag can transmit its data or not. If a tag has ASC which is the same value as PSC, the tag can transmit.

The tag has three states as follows: 1. Wait state: If the tag has ASC greater than PSC, it waits for other commands of reader. 2. Active state: If the tag has ASC equal to PSC, it transmits its data to the reader. 3. Sleep state: If the tag has ASC less than PSC, the tag does not transmit any data. This tag waits for next identification cycle because it has already been identified in current identification cycle.

In the collided slot, the colliding tags, the tags of the active state, add a random number ( 0 or 1$)$ to ASC. The active tags which select number 1 convert their state into wait state. The wait tags increase ASC, when collision occurs. When the reader send the message which means the idle slot to tags, the tags in the wait state decrease ASC.

Fig. 3(b) shows the operation of the adaptive binary splitting protocol. If the population of tag does not change, no collision and no idle occurs in current cycle under ABS scheme. The reader remembers the number of tags in previous cycle and informs tags of it at the start of identification cycle. If there are incoming tags, those tags randomly set their counter into smaller value than the number sent by the reader. Incoming tags can cause some collision with existing tags. When a collision occurs, those collided tags add a randomly selected binary number (0 or 1$)$ to ASC. If there are leaving tags, idle slot can occur. 


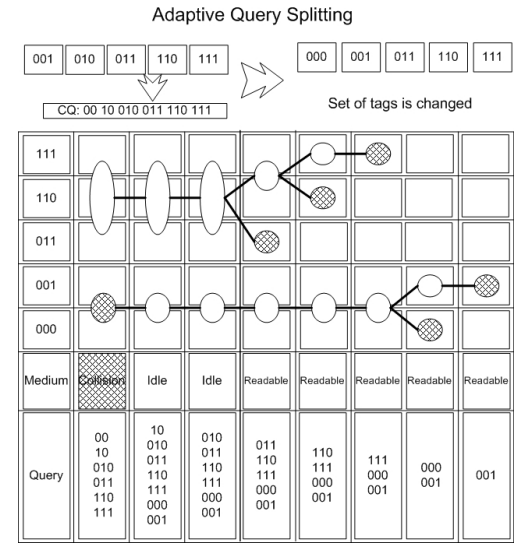

(a) adaptive query splitting protocol
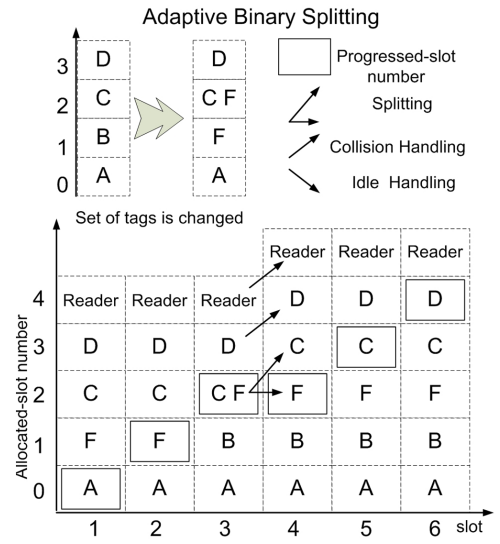

(b) adaptive binary splitting protocol

Fig. 3. The adaptive splitting protocols

\section{Performance Metrics}

RFID applications have various requirements. Mobility of tags is a key factor for branching properties of RFID applications. Mobility of tags varies according as what kinds of RFID applications are employed. In this section, we divide RFID applications into two cases. The first one is the case that has little mobility of tags. The second one is the case in which we have to consider mobility of tags for performing identification operation. In both case, we present some points of reference for evaluating performance of RFID tag anti-collision protocols.

\subsection{Total Identification Delay}

For some applications, one can locate objects in front of a reader, which then are identified. Such a scenario falls under where a reader is deployed at an entrance or an exit at which the products are disposed of. In this case, a user should determine the starting point and wait until all tags are identified. In these kinds of applications, the time for identifying all tags in reader's range is a critical factor for evaluating performance of RFID tag anti-collision protocols.

\subsection{The Number of Identified Tags}

We can consider the situation where objects with a RFID tag moves toward a reader through a conveyer belt. Under the situation of this kind, the relation between the velocity of conveyer belt and the number of identified tags can be considered as important guideline to evaluate the performance of tag anticollision protocols. The velocity of conveyer belt means the variation of tag 
population. If a tag anti-collision protocol can identify many tags under the situation that tags go through the reader's range fast, it means that the protocol can fast overcome the variation of tag set.

\subsection{Re-identification Interval}

In case a RFID reader has to recognize tags in reader's range persistently, a reader continues to perform identification process. The shorter the length of identification cycle is, the frequenter tags are identified. If a anti-collision protocol can access same tag many times, that protocol can recognize fast whether the tag is in reader's range or not. This can be described as re-identification interval and be an important factor for evaluating performance of RFID tag anti-collision protocols. The tag tracing can be performed precisely and quickly with decreasing the re-identification interval.

\section{Performance Evaluation}

In this section, we evaluate the performance of tag anti-collision protocols examined so far. We make following assumptions for reflecting only the effect on the operational principle of tag anti-collision protocols. Reliability in transmission between a reader and a tag is perfectly guaranteed. All the protocols consider make use of the same type of physical functionalities. All protocols have same time for transmitting their ID codes. The reader just targets tag identification and does not perform any additional operations.

The transmitted message format is set based on ISO 18000-6 specification. Fig. 4 describes data transmission between a reader and a tag. Since we would like to evaluate the performance of protocols based on transmission slots, we does not consider the computation delay between end point of data receiving and start point of data sending.

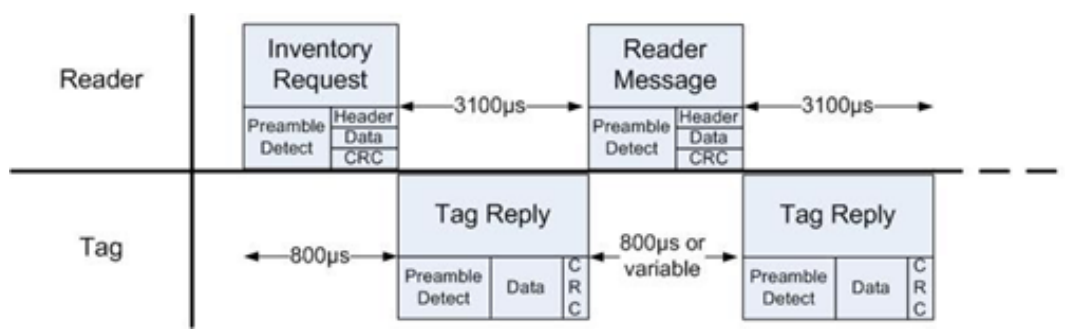

Fig. 4. Data transmission between a reader and a tag

\subsection{Total Identification Delay}

We perform simulation study to investigate how long a user should wait for at each protocol. As we assume that mobility of tags is not considered, a reader 


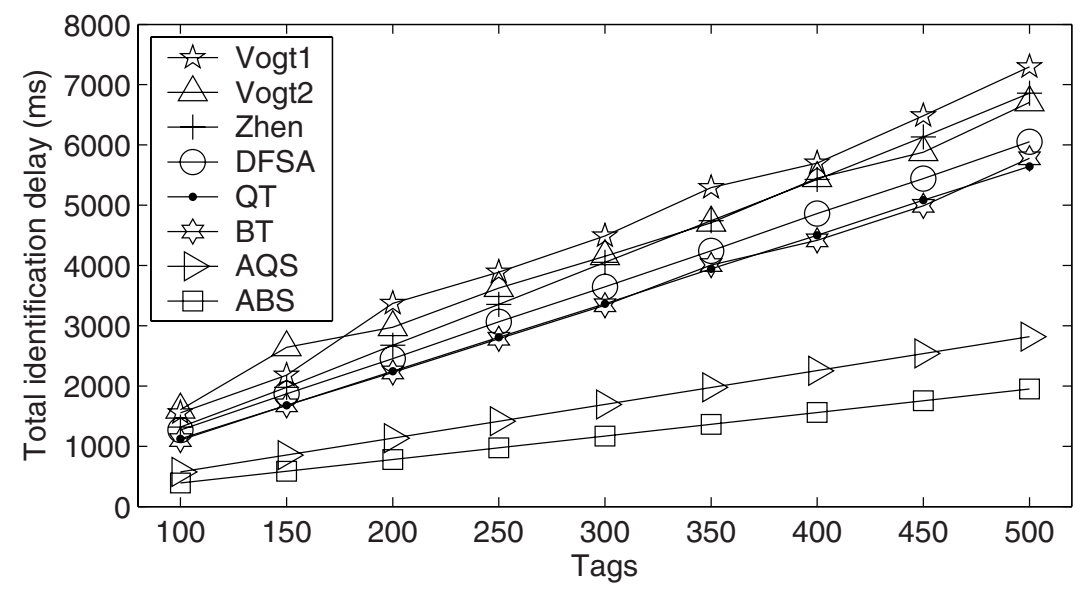

Fig. 5. Total identification delay

makes identification process without changes on tags sample during its identification.

Fig. 5 shows the relationship between the number of tags and the time taken to identify all tags by each protocol. Probabilistic tag anti-collision schemes are independent of each round, and keep three slots: the success, the readable, and the idle slot, all of which follows identical probabilistic distribution.

QT and BT, which belongs to the deterministic scheme, shows better performance than the probabilistic one. They are, however, little different in total identification delay. There is not much different in performance between probabilistic schemes until the samples are over 250. The reason why Vogts method becomes debased when samples over 300 is that it assumes the case where the maximum frame size is 256. In Zhen and DFSA, the predicted number of tags is employed as the frame size, so it outperforms Vogts when the number of tags increases. It is expected that both schemes can show the similar results provided that Vogts configure the frame size to the tags predicted. ABS and AQS experience the least delay out of the protocols. This is because they have already known information of the population of tags. That is, the simulation on them is performed after the identification is done once. Due to the inherent characteristics of ABS and AQS, under the same condition with BT and QT, we obtain the same results shown in them.

\subsection{The Number of Identified Tags}

This section presents performance of tag antic-collision schemes in the applications with high mobility of tags. We consider the situation where objects with a RFID tag moves toward a reader through a conveyer belt. There can be tops 200 tags within its readers range. We measured performance varying the velocity of a conveyer belt. 


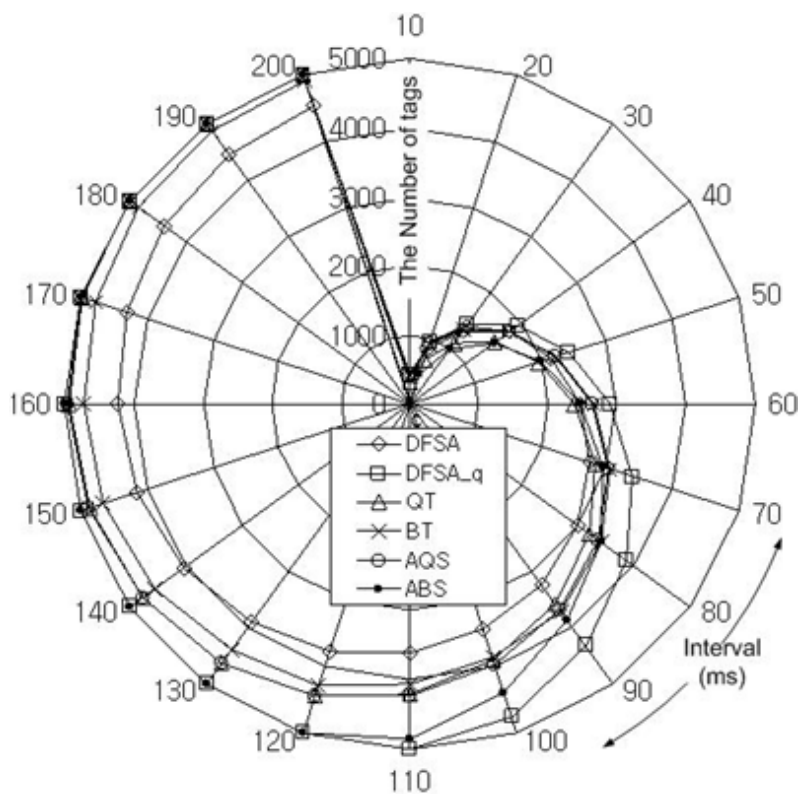

Fig. 6. The number of identified tags

Fig. 6 shows the identified number of objects at each anti-collision scheme when 5,000 objects get moved. The main purpose of this application aims to identify tags passing by a reader. In such an application, if tags are waken up in advance before identification process by a reader, we can have the advantage of adapting Quiet state of tags because the tag having been already identified does not need to perform that process again. We evaluate DFSA and DFSA_q (Quiet state) in Fig. 6.

As the speed of conveyer belt increases, the interval entering a readers range is reduced. In other words, the larger the interval increases, the slower the conveyer belt moves. DFSA using Quiet state succeed in identifying all tags of 5,000 even at the fastest condition. ABS is the second to them in adaptability of the speed of a conveyer belt.

The Probabilistic tag anti-collision schemes adapting Quiet state of tags outperforms since the number of tags is small in the initial part. The reader performs the identification operation with small number of tags and makes them sleep. In this case, the size of population is maintained at small size. On the other hand, because the tags which were already identified in pervious cycle rejoin the identification operation in current cycle in the deterministic tag anti-collision schemes, every cycle has more tags than the probabilistic tag anti-collision schemes adapting Quiet state.

Probabilistic tag anti-collision schemes without using the Quiet state of the tag cannot identify 5000 tags with $30 \mathrm{~ms}$ interval due to rejoining of identified tags. AQS and query tree have similar performance and the performance of the 


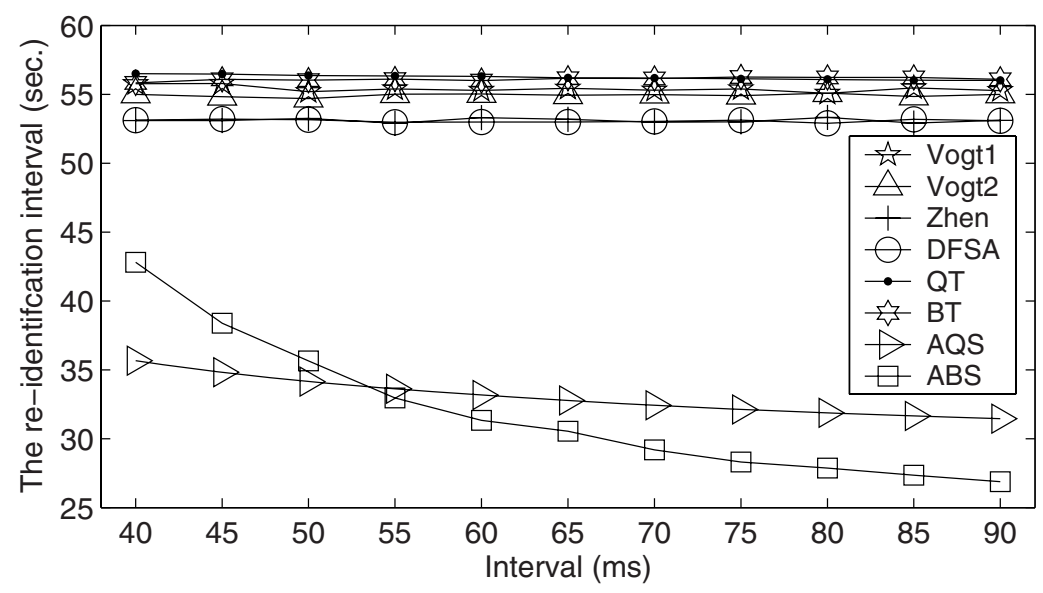

Fig. 7. Re-identification interval

binary tree is not good. However binary tree can identify all tags under the value of interval 0.026 per an incoming tag.

\subsection{Re-identification Interval}

We already define re-identification interval. As the application to make this metric be meaningful, we can consider the one where the state of the tags should be checked out in real-time. It is meaningless to use the Quiet state in such applications. It must be considered that the method to wake tag up in an appropriate timing. Because there has little investigated on such a method, we just compare the probabilistic tag anti-collision schemes without Quiet state with the deterministic ones.

In a conveyer belt, the interval and the moving speed of the tag can be regarded as the speed of the belt and the rate of changes of samples, respectively. Fig. 7 shows the re-identification interval per unit time. Given the interval is over $40 \mathrm{~ms}$, since even probabilistic schemes without Quiet state achieves high identification ratio over $98 \%$, we depict the results from $40 \mathrm{~ms}$ in interval. ABS and AQS shows desirable results, while the other schemes are even. ABS and AQS achieves good performance than other schemes because they can take advantage of the identification information of the previous stage.

\section{Conclusion}

In this paper, we have introduced tag anti-collision schemes and evaluated the performance of them. According to the types of RFID applications, we considered two cases: motionless tags and moving tags. For applications employing motionless tags, a user can determine the start and end of the identification process. When such applications are considered, probabilistic and deterministic 
tag anti-collision schemes show the similar ability in identifying tags. For applications requiring persistent observations on tags, AQS and ABS outperform any other schemes, especially under the situation that tag population varies at low speed. An important factor we should consider in evaluating performance of persistent observation is to identify whether a tag exists within its reader's range as quickly as possible. We also show that AQS and ABS are appropriate to meet this evaluating factor.

\section{Acknowledgement}

This work was supported by the Korea Science and Engineering Foundation (KOSEF) grant funded by the Korea government (MOST) (No. R01-2007-00011203-0).

\section{References}

1. Finkenzeller, K.: RFID Handbook: Fundamentals and Applications in Contactless Smart Cards and Identification. John Wiley \& Sons, Chichester (2003)

2. EPCTM Radio-Frequency Identification Protocols Class-1 Generation-2 UHF RFID Protocol for Communications at $860 \mathrm{MHz}-960 \mathrm{MHz}$ Version 1.0.8, EPCglobal (December 2004)

3. Information Technology Automatic Identification and Data Capture Techniques Radio Frequency Identification for Item Management Air Interface - Part 6: Parameters for Air Interface Communications at 860-960 MHz, Final Draft International Standard ISO 18000-6.

4. Vogt, H.: Efficient Object Identification with Passive RFID Tags. In: Proc. of the International Conference on Pervasive Computing, pp. 98-113 (April 2002)

5. Cha, J., Kim, J.: Dynamic Framed Slotted ALOHA Algorithm Using Fast Tag Estimation Method for RFID System. In: Cha, J., Kim, J. (eds.) Proc. of the IEEE Consumer Communications and Networking Conference, Las Vegas, USA (January 2006)

6. Zhen, B., Kobayashi, M., Shimizu, M.: Framed ALOHA for multiple RFID objects identification. IEICE Transactions on Communications E88-B(3), 991-999 (2005)

7. Law, C., Lee, K., Siu, K.-Y.: Efficient memoryless protocol for tag identification. In: Proc. International Workshop on Discrete Algorithms and Methods for Mobile Computing and Communications, pp. 75-84 (2000)

8. Myung, J., Lee, W.: An adaptive memoryless tag anti-collision protocol for RFID networks. In: Proc. IEEE INFOCOM (2005)

9. Myung, J., Lee, W.: Adaptive Splitting Protocols for RFID Tag Collision Arbitration. In: Proc. ACM MOBIHOC, pp. 202-213 (2006) 